AGRICULTURE AND BIOLOGY JOURNAL OF NORTH AMERICA

ISSN Print: 2151-7517, ISSN Online: 2151-7525, doi:10.5251/abjna.2012.3.12.486.492

(C) 2012, ScienceHuß, http://www.scihub.org/ABJNA

\title{
Preliminary assessment of tree species diversity in Afi Mountain Wildlife Sanctuary, Southern Nigeria
}

\author{
${ }^{1}$ Daniel I. Edet, ${ }^{1}$ Henry M. Ijeomah and ${ }^{2}$ Augustine U. Ogogo \\ ${ }^{1}$ Department of Forestry and Wildlife Management, University of Port Harcourt, P.M.B 5323, \\ Choba, Port Harcourt, Nigeria \\ E-mail address of corresponding author: daniffer2003@yahoo.com \\ ${ }^{2}$ Department of Forestry and Wildlife Resources Management \\ University of Calabar, P.M.B 1115 Calabar, Nigeria.
}

\begin{abstract}
Appraisal of flora species which form an integral part of animal ecology, in wildlife based protected areas is necessary before any meaningful conservation work can commence. This informed the study on the preliminary assessment of tree species diversity in Afi Mountain Wildlife Sanctuary (AMWS) and its adjoining communal forest, a biodiversity hotspot in Nigeria. The Point Centre Quarter Method (PCQM) was employed for the study while the Shannon-Weiner Diversity Index $(H)$ and student $t$ test were used to analyse data generated. The study revealed a total of 102 tree species in 35 families and 74 tree species in 31 families for the protected (sanctuary) and communal forests respectively. Afzelia bipidensis $(R D=5.00)$ and Ceiba pentandra $(R D=$ 7.50) were the abundant species in the protected and communal forests respectively. Forty-nine (49) and twenty-seven (27) tree species were recorded as threatened in the protected and communal forests respectively. The Shannon-Weiner Diversity Indices obtained for the sanctuary $\left(H_{1}=4.3459\right)$ and communal forest $\left(H_{2}=4.0250\right)$ are not significantly different $(p>0.05)$. The dominance of Ceiba pentandra and presence of Harungana madagascariensis, and Musanga cecropioides in the communal forest indicated a characteristic of a secondary forest occasioned by anthropogenic impacts.
\end{abstract}

Keywords: Assessment; tree species; diversity indices; sanctuary; communal forest.

\section{INTRODUCTION}

The Afi Mountain Wildlife Sanctuary (AMWS) was established to protect a significant portion of the Afi River Forest Reserve in Cross River State, Nigeria, which has suffered severe anthropogenic impacts for decades. This became necessary in view of the fact that the sanctuary is a biodiversity hotspot characterized by species diversity and endemism (Conservation International, 2005). The area has suffered from activities of illegal logging operators thereby threatening important flora and fauna species (Agbor, 2003). Thus there is need to appraise the composition of tree species using diversity indices to ascertain the present status of tree species which form an integral part of wild animal habitat of the sanctuary.

It is quiet obvious that data generated from the study will promote effective conservation of biodiversity within the sanctuary and adjoining communal forest. The objective of the study is therefore to quantify tree species composition and abundance using diversity indices in Afi Mountain Wildlife Sanctuary and its adjoining communal forests.

\section{MATERIALS AND METHODS}

AREA OF STUDY: The area of study is Afi Mountain Wildlife Sanctuary (AMWS) and its adjoining communal forests. The Sanctuary is situated within the mountainous and relatively rugged rainforest block in the border region of South-Eastern Nigeria and South-West Cameroon, an area recognized as one of Africa's biodiversity hotspots (Conservation International, 2005). The sanctuary which is located within the Afi River Forest Reserve in Boki Local Government Area of Cross River State, Nigeria, lies approximately between latitude $6^{\circ} 1^{\prime}$ and $6^{\circ} 25^{\prime}$ North and between longitude $8^{\circ} 55^{\prime}$ and $9^{\circ}$ 15' East. Following renewed international attention in the late 1980s, the creation of a wildlife sanctuary at Afi Mountains for the conservation of the endemic Cross River Gorilla and other wildlife species was recommended. Thus in May 2000, the Cross River State Government gazetted the Afi Mountain Wildlife 
Sanctuary covering approximately $104 \mathrm{~km}^{2}$ (Wikipedia, 2012)

The Afi Mountain Wildlife Sanctuary falls within the tropical high forest vegetation zone. The entire area falls within a broad annual rain fall zone of $3,000 \mathrm{~mm}$ - 3,800 mm but, with a variation increasing from lowland to uphill (Agbor, 2003). Rainy season starts around late March / early April to September with a break in August. The dry season starts from October and ends in March. The mean monthly maximum temperature ranges from $22.2^{\circ} \mathrm{C}$ to $27.4^{\circ} \mathrm{C}$ (Edet, 2010).

Notable endemic and endangered species of wildlife include the Cross River gorilla (Gorilla gorilla diehli), Nigeria chimpanzee (Pan troglodytes vellorosus) and drill (Mandrillus leucophaeus). Other known wildlife species which are protected by the Endangered Species Decree 11 of 1985 include the red-eared guenon (Cercopithecus erythrotis), mona guenon (Cercopithecus mona) and red river hog (Potamochoerus porcus). Presently, the sanctuary habours the world's largest roosting site for migrating European barn swallows (Hirundo rustica), and also an important nesting site for the rare bare-necked rock fowl (Picarthertes oreas) (Edet, 2010).

\section{MATERIALS USED FOR THE STUDY}

The materials used for the study include measuring tapes $(50 \mathrm{~m})$, point centre quadrant, twine, ranging Poles, machetes and flagging tapes.

METHODOLOGY: The Point Centre Quarter Method (PCQM) as described by Bryant et al. (2005) was employed to assess tree species composition in Afi Mountain Wildlife Sanctuary and its adjoining communal forest in December, 2011

Fifteen (15) transects of $1 \mathrm{~km}(1000 \mathrm{~m})$ each in length were cut in each of the survey area (i.e. the sanctuary and communal forest). Out of the fifteen (15) transects, five (5) were randomly selected in each survey area. This gave a total of $5 \mathrm{~km}(5000 \mathrm{~m})$ length of transects in each survey area. A communal forest belonging to Katabang (an adjoining community to AMWS) was selected for this study. Transects were pegged at 100 metres interval and the point centre quadrant dropped systematically at this point. This gave a total of 50 sampling points with a record of 200 trees per sampled area. Only trees of $1 \mathrm{~m}$ and above in height nearest to the sampling point in each quarter of the quadrant were enumerated and recorded.

\section{DATA ANALYSES}

Data obtained from tree species composition was analysed to obtain relative density (\%) and relative abundance.

$\begin{aligned} & \text { Relative density of species } \\ & \text { Number of individual species }\end{aligned} \times 100$
Total number of trees
$\frac{\text { Relative abundance of species }}{\text { Relative density of species }}$
100

The various species were scored according to their relative densities (RD); i.e. abundant ( $R D \geq 5.00$ ), frequent $(4.00 \leq \mathrm{RD} \leq 4.99)$, occasional $(3.00 \leq \mathrm{RD} \leq$ $3.99)$, rare $(1.00 \leq R D \leq 2.99)$ and threatened / endangered $(0.00<\mathrm{RD} \leq 1.00)$

Data obtained from relative abundance was used to compute the Shannon - Wiener's Diversity Index $(H)$ as described by Stirn (1981) for the protected and unprotected (communal) forests of the area. Shannon-Wiener's Diversity Index in given by the equation

$H=-\sum_{i=1}^{s} P i \ln P i$

Where $H=$ Shannon-Wiener's Diversity Index, $P_{i}=$ Relative abundance of the $i^{\text {th }}$ species, $\ln P_{i}=$ Natural logarithm of the corresponding relative abundance $\left(P_{i}\right)$ of the species. The values of Shannon - Wiener Index obtained from the two locations were compared using the student $t$ test as described by Jayaraman (1999). The $t$ test is given by the equation:

$t=\frac{\left|H_{1}-H_{2}\right|}{\sqrt{\operatorname{Var}\left(H_{1}\right)+\operatorname{Var}\left(H_{2}\right)}}$

$v=\frac{\left[\operatorname{Var}\left(H_{1}\right)+\operatorname{Var}\left(H_{2}\right)\right]^{2}}{\left[\operatorname{Var}\left(H_{1}\right)\right]^{2} /{ }_{N_{1}}+\left[\operatorname{Var}\left(H_{2}\right)\right]^{2} / N_{N_{2}}}$

as:

where

$\operatorname{Var}(H)=\frac{\sum P i(\ln P i)^{2}-\left(\sum P i \ln P i\right)^{2}}{N}+\frac{S-1}{2 N^{2}}$

$H_{1}=$ Shannon - Wiener Index value obtained for the protected forest, $\mathrm{H}_{2}=$ Shannon - Wiener Index value obtained for the communal forest, $\operatorname{Var}(H)=$ Variance in diversity for each location, $\mathrm{S}=$ Number of species present per location, $\mathrm{N}=$ Number of trees counted per location. 
Agric. Biol. J. N. Am., 2012, 3(12): 486-492

\section{RESULTS}

Table 1: Indices of Tree species composition in protected forest of AMWS

\begin{tabular}{|c|c|c|c|c|c|c|c|}
\hline Family & Species & $\mathrm{RD}$ & $P_{i}$ & $\ln P_{i}$ & $P_{i}\left(\ln P_{i}\right)$ & $(\ln P I)^{2}$ & $P i(\operatorname{InPl})^{2}$ \\
\hline Annacardiaceae & Antrocaryon klaineanum & 0.50 & 0.005 & -5.2983 & -0.0265 & 28.0720 & 0.1404 \\
\hline Annacardiaceae & Antrocaryon micaster & 0.50 & 0.005 & -5.2983 & -0.0265 & 28.0720 & 0.1404 \\
\hline Annacardiaceae & Sorindeia mildbraedii & 0.50 & 0.005 & -5.2983 & -0.0265 & 28.0720 & 0.1404 \\
\hline Annacardiaceae & Spondias mombin & 1.00 & 0.010 & -4.6052 & -0.0461 & 21.2079 & 0.2121 \\
\hline Anisophyliaceae & Poga oleosa & 0.50 & 0.005 & -5.2983 & -0.0265 & 28.0720 & 0.1404 \\
\hline Annonaceae & Monodora myristica & 0.50 & 0.005 & -5.2983 & -0.0265 & 28.0720 & 0.1404 \\
\hline Annonaceae & Xylopia Africana & 1.00 & 0.010 & -4.6052 & -0.0461 & 21.2079 & 0.2121 \\
\hline Annonaceae & Xylopia staudtia & 0.50 & 0.005 & -5.2983 & -0.0265 & 28.0720 & 0.1404 \\
\hline Apocynaceae & Alstonia boonei & 1.00 & 0.010 & -4.6052 & -0.0461 & 21.2079 & 0.2121 \\
\hline Apocynaceae & Alstonia congensis & 1.50 & 0.015 & -4.1997 & -0.0630 & 17.6375 & 0.2646 \\
\hline Apocynaceae & Funtumia elastic & 1.50 & 0.015 & -4.1997 & -0.0630 & 17.6375 & 0.2646 \\
\hline Apocynaceae & Rauvolfia vomitoria & 0.50 & 0.005 & -5.2983 & -0.0265 & 28.0720 & 0.1404 \\
\hline Apocynaceae & Pleiocarpa talbotii & 0.50 & 0.005 & -5.2983 & -0.0265 & 28.0720 & 0.1404 \\
\hline Bignoniaceae & Newbouldia laevis & 1.00 & 0.010 & -4.6052 & -0.0461 & 21.2079 & 0.2121 \\
\hline Bombacaceae & Ceiba pentandra & 2.50 & 0.025 & -3.6889 & -0.0922 & 13.6080 & 0.3402 \\
\hline Bombaceae & Bombax buonopozense & 1.00 & 0.010 & -4.6052 & -0.0461 & 21.2079 & 0.2121 \\
\hline Buseraceae & Canarium schweinfurthii & 1.50 & 0.015 & -4.1997 & -0.0630 & 17.6375 & 0.2646 \\
\hline Buseraceae & Dacryodes edulis & 1.00 & 0.010 & -4.6052 & -0.0461 & 21.2079 & 0.2121 \\
\hline Connaraceae & Cnetis ferruginea & 0.50 & 0.050 & -5.2983 & -0.0265 & 28.0720 & 0.1404 \\
\hline Combretaceae & Terminalia ivorensis & 1.00 & 0.010 & -4.6052 & -0.0461 & 21.2079 & 0.2121 \\
\hline Combretaceae & Terminalia superb & 1.00 & 0.010 & -4.6052 & -0.0461 & 21.2079 & 0.2121 \\
\hline Ebenaceae & Diospyros heudelotii & 0.50 & 0.005 & -5.2983 & -0.0265 & 28.0720 & 0.1404 \\
\hline Ebenaceae & Diospyros melocarpa & 1.00 & 0.010 & -4.6052 & -0.0461 & 21.2079 & 0.2121 \\
\hline Ebenaceae & Diospyros zenkerii & 0.50 & 0.005 & -5.2983 & -0.0265 & 28.0720 & 0.1404 \\
\hline Euphorbiaceaea & Alchornia laxiflora & 0.50 & 0.005 & -5.2983 & -0.0265 & 28.0720 & 0.1404 \\
\hline Euphorbiaceaea & Anthonota fragrance & 0.50 & 0.005 & -5.2983 & -0.0265 & 28.0720 & 0.1404 \\
\hline Euphorbiaceaea & Cyrtogonne argentia & 0.50 & 0.005 & -5.2983 & -0.0265 & 28.0720 & 0.1404 \\
\hline Euphorbiaceaea & Grosseria vignei & 0.50 & 0.005 & -5.2983 & -0.0265 & 28.0720 & 0.1404 \\
\hline Euphorbiaceaea & Maesobotrya dusenii & 1.00 & 0.010 & -4.6052 & -0.0461 & 21.2079 & 0.2121 \\
\hline Euphorbiaceaea & Maesobotrya staudtii & 0.50 & 0.005 & -5.2983 & -0.0265 & 28.0720 & 0.1404 \\
\hline Euphorbiaceaea & Rhicinodendron heudelotii & 1.00 & 0.010 & -4.6052 & -0.0461 & 21.2079 & 0.2121 \\
\hline Euphorbiaceaea & Uapaca acuminate & 0.50 & 0.005 & -5.2983 & -0.0265 & 28.0720 & 0.1404 \\
\hline Flacourtiaceae & Compostylus ovalis & 0.50 & 0.005 & -5.2983 & -0.0265 & 28.0720 & 0.1404 \\
\hline Flacourtiaceae & Ophiobostyrs zenkerii & 0.50 & 0.005 & -5.2983 & -0.0265 & 28.0720 & 0.1404 \\
\hline Guttiferae & Garcinia kola & 1.50 & 0.015 & -4.1997 & -0.0630 & 17.6375 & 0.2646 \\
\hline Guttiferae & Garcinia manni & 1.00 & 0.010 & -4.6052 & -0.0461 & 21.2079 & 0.2121 \\
\hline Guttiferae & $\begin{array}{l}\text { Harungana } \\
\text { madagascariensis }\end{array}$ & 0.50 & 0.005 & -5.2983 & -0.0265 & 28.0720 & 0.1404 \\
\hline Guttiferae & Mammea africanum & 1.00 & 0.010 & -4.6052 & -0.0461 & 21.2079 & 0.2121 \\
\hline Irvingiaceae & Irvingia gabonensis & 1.00 & 0.010 & -4.6052 & -0.0461 & 21.2079 & 0.2121 \\
\hline Irvingiaceae & Irvingia wombulu & 0.50 & 0.005 & -5.2983 & -0.0265 & 28.0720 & 0.1404 \\
\hline Leguminosae & Afzelia bipidensis & 5.00 & 0.050 & -2.9957 & -0.1498 & 8.9742 & 0.4487 \\
\hline Leguminosae & Albizia ferruginea & 1.50 & 0.015 & -4.1997 & -0.0630 & 17.6375 & 0.2646 \\
\hline Leguminosae & Albizia lebbeck & 0.50 & 0.005 & -5.2983 & -0.0265 & 28.0720 & 0.1404 \\
\hline Leguminosae & Albizia zygia & 1.00 & 0.010 & -4.6052 & -0.0461 & 21.2079 & 0.2121 \\
\hline Leguminosae & Angylocalyx oligophyllus & 0.50 & 0.005 & -5.2983 & -0.0265 & 28.0720 & 0.1404 \\
\hline Leguminosae & Brachystegia eurycoma & 1.50 & 0.015 & -4.1997 & -0.0630 & 17.6375 & 0.2646 \\
\hline Leguminosae & Daniella ogea & 1.50 & 0.015 & -4.1997 & -0.0630 & 17.6375 & 0.2646 \\
\hline Leguminosae & Dialum guineense & 1.50 & 0.015 & -4.1997 & -0.0630 & 17.6375 & 0.2646 \\
\hline Leguminosae & Parkia bicolor & 2.50 & 0.025 & -3.6889 & -0.0922 & 13.6080 & 0.3402 \\
\hline Leguminosae & Pentaclethra macrophylla & 1.00 & 0.010 & -4.6052 & -0.0461 & 21.2079 & 0.2121 \\
\hline Leguminosae & $\begin{array}{l}\text { Piptandeniastrum } \\
\text { africanum }\end{array}$ & 1.50 & 0.015 & -4.1997 & -0.0630 & 17.6375 & 0.2646 \\
\hline
\end{tabular}


Agric. Biol. J. N. Am., 2012, 3(12): 486-492

\begin{tabular}{|c|c|c|c|c|c|c|c|}
\hline Leguminosae & Pterocarpus erinaceous & 1.00 & 0.010 & -4.6052 & -0.0461 & 21.2079 & 0.2121 \\
\hline Leguminosae & Pterocarpus mildbraedii & 0.50 & 0.005 & -5.2983 & -0.0265 & 28.0720 & 0.1404 \\
\hline Leguminosae & Pterocarpus osun & 0.50 & 0.005 & -5.2983 & -0.0265 & 28.0720 & 0.1404 \\
\hline Leguminosae & Pterocarpus soyauxii & 0.50 & 0.005 & -5.2983 & -0.0265 & 28.0720 & 0.1404 \\
\hline Leguminosae & Tetrapleura tetraptera & 2.50 & 0.025 & -3.6889 & -0.0922 & 13.6080 & 0.3402 \\
\hline Leguminosae & Zenkerella citran & 1.00 & 0.010 & -4.6052 & -0.0461 & 21.2079 & 0.2121 \\
\hline Loganiaceae & Anthocleista djalonensis & 0.50 & 0.005 & -5.2983 & -0.0265 & 28.0720 & 0.1404 \\
\hline Meliaceae & $\begin{array}{l}\text { Entandrophragma } \\
\text { angolense }\end{array}$ & 0.50 & 0.005 & -5.2983 & -0.0265 & 28.0720 & 0.1404 \\
\hline Meliaceae & $\begin{array}{l}\text { Entandrophragma } \\
\text { cylindricum }\end{array}$ & 1.00 & 0.010 & -4.6052 & -0.0461 & 21.2079 & 0.2121 \\
\hline Meliaceae & Guarea glomerulata & 0.50 & 0.005 & -5.2983 & -0.0265 & 28.0720 & 0.1404 \\
\hline Meliaceae & Khaya grandifolia & 1.00 & 0.010 & -4.6052 & -0.0461 & 21.2079 & 0.2121 \\
\hline Meliaceae & Khaya ivorensis & 1.00 & 0.010 & -4.6052 & -0.0461 & 21.2079 & 0.2121 \\
\hline Meliaceae & Lovoa trichiloides & 0.50 & 0.005 & -5.2983 & -0.0265 & 28.0720 & 0.1404 \\
\hline Meliaceae & Melicea excels & 1.50 & 0.015 & -4.1997 & -0.0630 & 17.6375 & 0.2646 \\
\hline Meliaceae & Melicea zygia & 0.50 & 0.005 & -5.2983 & -0.0265 & 28.0720 & 0.1404 \\
\hline Mimosaceae & Newtonia duparquetiana & 0.50 & 0.005 & -5.2983 & -0.0265 & 28.0720 & 0.1404 \\
\hline Moraceae & Anthodeista vogelii & 0.50 & 0.005 & -5.2983 & -0.0265 & 28.0720 & 0.1404 \\
\hline Moraceae & Musanga cercropioides & 1.00 & 0.010 & -4.6052 & -0.0461 & 21.2079 & 0.2121 \\
\hline Moraceae & Myriathus arboreus & 0.50 & 0.005 & -5.2983 & -0.0265 & 28.0720 & 0.1404 \\
\hline Moraceae & Treculia Africana & 2.00 & 0.020 & -3.9120 & -0.0782 & 15.3037 & 0.3061 \\
\hline Myristicaceae & Pycnanthus angolensis & 1.00 & 0.010 & -4.6052 & -0.0461 & 21.2079 & 0.2121 \\
\hline Myristicaceae & $\begin{array}{l}\text { Pycnanthus } \\
\text { microcephalus }\end{array}$ & 0.50 & 0.005 & -5.2983 & -0.0265 & 28.0720 & 0.1404 \\
\hline Ochnaceae & Lophira alata & 2.00 & 0.020 & -3.9120 & -0.0782 & 15.3037 & 0.3061 \\
\hline Palmae & Elaeis guineensis & 1.00 & 0.010 & -4.6052 & -0.0461 & 21.2079 & 0.2121 \\
\hline Papilionaceae & Baphia nitida & 0.50 & 0.005 & -5.2983 & -0.0265 & 28.0720 & 0.1404 \\
\hline Polygalaceae & Carpolobia alba & 0.50 & 0.005 & -5.2983 & -0.0265 & 28.0720 & 0.1404 \\
\hline Polygalaceae & Carpolobia lutea & 1.00 & 0.010 & -4.6052 & -0.0461 & 21.2079 & 0.2121 \\
\hline Polygonaceae & Antiaris Africana & 2.50 & 0.025 & -3.6889 & -0.0922 & 13.6080 & 0.3402 \\
\hline Rubiaceae & Didymosalpinx parviflora & 0.50 & 0.005 & -5.2983 & -0.0265 & 28.0720 & 0.1404 \\
\hline Rubiaceae & Heinsia crinata & 1.00 & 0.010 & -4.6052 & -0.0461 & 21.2079 & 0.2121 \\
\hline Rubiaceae & Hymenodictyon biafranum & 0.50 & 0.005 & -5.2983 & -0.0265 & 28.0720 & 0.1404 \\
\hline Rubiaceae & Massularia acuminate & 0.50 & 0.005 & -5.2983 & -0.0265 & 28.0720 & 0.1404 \\
\hline Rubiaceae & Mytragyna stipulosa & 1.50 & 0.015 & -4.1997 & -0.0630 & 17.6375 & 0.2646 \\
\hline Rubiaceae & Nauclea diderrichi & 1.00 & 0.010 & -4.6052 & -0.0461 & 21.2079 & 0.2121 \\
\hline Rosaceae & Parinari chrysophylla & 0.50 & 0.005 & -5.2983 & -0.0265 & 28.0720 & 0.1404 \\
\hline Rutaceae & Tectea afzeli & 0.50 & 0.005 & -5.2983 & -0.0265 & 28.0720 & 0.1404 \\
\hline Rutaceae & $\begin{array}{l}\text { Zanthoxylum } \\
\text { xanthoxyloides }\end{array}$ & 1.50 & 0.015 & -4.1997 & -0.0630 & 17.6375 & 0.2646 \\
\hline Sapindaceae & Blighia sapida & 1.00 & 0.010 & -4.6052 & -0.0461 & 21.2079 & 0.2121 \\
\hline Sapindaceae & Placodiscus tubiniatus & 0.50 & 0.005 & -5.2983 & -0.0265 & 28.0720 & 0.1404 \\
\hline Sapotaceae & Bailonella toxisperma & 0.50 & 0.005 & -5.2983 & -0.0265 & 28.0720 & 0.1404 \\
\hline Sapotaceae & Chrysophylum albidum & 1.50 & 0.015 & -4.1997 & -0.0630 & 17.6375 & 0.2646 \\
\hline Sterculiaceae & Cola gigantean & 0.50 & 0.005 & -5.2983 & -0.0265 & 28.0720 & 0.1404 \\
\hline Sterculiaceae & Cola lepidota & 1.00 & 0.010 & -4.6052 & -0.0461 & 21.2079 & 0.2121 \\
\hline Sterculiaceae & Cola millenii & 1.00 & 0.010 & -4.6052 & -0.0461 & 21.2079 & 0.2121 \\
\hline Sterculiaceae & Cola pachycarpa & 0.50 & 0.005 & -5.2983 & -0.0265 & 28.0720 & 0.1404 \\
\hline Sterculiaceae & Leptobychia pallid & 0.50 & 0.005 & -5.2983 & -0.0265 & 28.0720 & 0.1404 \\
\hline Sterculiaceae & Sterculia tragacantha & 1.00 & 0.010 & -4.6052 & -0.0461 & 21.2079 & 0.2121 \\
\hline Sterculiaceae & Triplochiton scleroxylon & 2.00 & 0.020 & -3.9120 & -0.0782 & 15.3037 & 0.3061 \\
\hline Styraceae & Afrostyrax lepidophyllus & 1.00 & 0.010 & -4.6052 & -0.0461 & 21.2079 & 0.2121 \\
\hline Tiliaceae & Deplatsia dewevrei & 0.50 & 0.005 & -5.2983 & -0.0265 & 28.0720 & 0.1404 \\
\hline Verbenaceae & Vitex doniana & 0.50 & 0.005 & -5.2983 & -0.0265 & 28.0720 & 0.1404 \\
\hline
\end{tabular}


Agric. Biol. J. N. Am., 2012, 3(12): 486-492

Table 2: Indices of Tree species composition in communal forests adjoining AMWS

\begin{tabular}{|c|c|c|c|c|c|c|c|}
\hline Family & Species & $\mathrm{RD}$ & $P_{i}$ & $\ln P_{i}$ & $P_{i}\left(\ln P_{i}\right)$ & $(\ln P I)^{2}$ & $P i(\ln P I)^{2}$ \\
\hline Annacardiaceae & Spondias mombin & 1.00 & 0.010 & -4.6052 & -0.0461 & 21.2079 & 0.2121 \\
\hline Anisophylleceae & Poga oleosa & 0.50 & 0.005 & -5.2983 & -0.0265 & 28.0720 & 0.1404 \\
\hline Annonaceae & Enanthia chlorantha & 2.00 & 0.020 & -3.9120 & -0.0782 & 15.3037 & 0.3061 \\
\hline Annonaceae & Monodora myristica & 2.00 & 0.020 & -3.9120 & -0.0782 & 15.3037 & 0.3061 \\
\hline Annonaceae & Xylopia Africana & 0.50 & 0.005 & -5.2983 & -0.0265 & 28.0720 & 0.1404 \\
\hline Annonaceae & Xylopia staudtia & 0.50 & 0.005 & -5.2983 & -0.0265 & 28.0720 & 0.1404 \\
\hline Apocynaceae & Alstonia boonei & 1.50 & 0.015 & -4.1997 & -0.0630 & 17.6375 & 0.2646 \\
\hline Apocynaceae & Alstonia congensis & 0.50 & 0.005 & -5.2983 & -0.0265 & 28.0720 & 0.1404 \\
\hline Apocynaceae & Funtumia elastica & 1.50 & 0.015 & -4.1997 & -0.0630 & 17.6375 & 0.2646 \\
\hline Apocynaceae & Rauvolfia vomotora & 0.50 & 0.005 & -5.2983 & -0.0265 & 28.0720 & 0.1404 \\
\hline Bombacaceae & Ceiba pentandra & 7.50 & 0.075 & -2.5903 & -0.1943 & 6.7097 & 0.5032 \\
\hline Bombaceae & Bombax buonopozense & 1.00 & 0.010 & -4.6052 & -0.0461 & 21.2079 & 0.2121 \\
\hline Bignoniaceae & Newbouldia leavis & 2.00 & 0.020 & -3.9120 & -0.0782 & 15.3037 & 0.3061 \\
\hline Buseraceae & Dacryodes edulis & 2.50 & 0.025 & -3.6889 & -0.0922 & 13.6080 & 0.3402 \\
\hline Combretaceae & Terminalia ivorensis & 0.50 & 0.005 & -5.2983 & -0.0265 & 28.0720 & 0.1404 \\
\hline Combretaceae & Terminalia superb & 1.00 & 0.010 & -4.6052 & -0.0461 & 21.2079 & 0.2121 \\
\hline Ebenaceae & Diospyros heudelotii & 1.00 & 0.010 & -4.6052 & -0.0461 & 21.2079 & 0.2121 \\
\hline Ebenaceae & Diospyros melocarpa & 0.50 & 0.005 & -5.2983 & -0.0265 & 28.0720 & 0.1404 \\
\hline Euphorbiaceae & Maesobotrya dusenii & 1.50 & 0.015 & -4.1997 & -0.0630 & 17.6375 & 0.2646 \\
\hline Euphorbiaceae & Rhicinodendron heudelotii & 1.00 & 0.010 & -4.6052 & -0.0461 & 21.2079 & 0.2121 \\
\hline Euphorbiaceae & Uapaca acuminate & 1.00 & 0.010 & -4.6052 & -0.0461 & 21.2079 & 0.2121 \\
\hline Guttiferae & Garcinia kola & 1.00 & 0.010 & -4.6052 & -0.0461 & 21.2079 & 0.2121 \\
\hline Guttiferae & $\begin{array}{l}\text { Harungana } \\
\text { madagascariensis }\end{array}$ & 4.50 & 0.045 & -3.1011 & -0.1395 & 9.6168 & 0.4328 \\
\hline Guttiferae & Mammea Africana & 0.50 & 0.005 & -5.2983 & -0.0265 & 28.0720 & 0.1404 \\
\hline Icacinaceae & Lasianthera Africana & 1.50 & 0.015 & -4.1997 & -0.0630 & 17.6375 & 0.2646 \\
\hline Irvingiaceae & Irvingia gabonensis & 1.50 & 0.015 & -4.1997 & -0.0630 & 17.6375 & 0.2646 \\
\hline Irvingiaceae & Irvingia wombulu & 0.50 & 0.005 & -5.2983 & -0.0261 & 28.0720 & 0.1404 \\
\hline Leguminosae & Afzelia Africana & 1.00 & 0.010 & -4.6052 & -0.0461 & 21.2079 & 0.2121 \\
\hline Leguminosae & Afzelia bipidensis & 2.50 & 0.025 & -3.6889 & -0.0922 & 13.6080 & 0.3402 \\
\hline Leguminosae & Abizia ferruginea & 0.50 & 0.005 & -5.2983 & -0.0265 & 28.0720 & 0.1404 \\
\hline Leguminosae & Albizia zygia & 4.50 & 0.045 & -3.1011 & -0.1395 & 9.6168 & 0.4328 \\
\hline Leguminosae & Angylocarlyx oligophyllus & 0.50 & 0.005 & -5.2983 & -0.0265 & 28.0720 & 0.1404 \\
\hline Leguminosae & Brachystegia eurycoma & 2.00 & 0.020 & -3.9120 & -0.0782 & 15.3037 & 0.3061 \\
\hline Leguminosae & Daniella ogea & 0.50 & 0.005 & -5.2983 & -0.0265 & 28.0720 & 0.1404 \\
\hline Leguminosae & Dialum guineense & 2.00 & 0.020 & -3.9120 & -0.0782 & 15.3037 & 0.3061 \\
\hline Leguminosae & Parkia bicolor & 2.50 & 0.025 & -3.6889 & -0.0922 & 13.6080 & 0.3402 \\
\hline Leguminosae & Pentaclethra macrophylla & 1.00 & 0.010 & -4.6052 & -0.0461 & 21.2079 & 0.2121 \\
\hline Leguminosae & Pterocarpus erinaceous & 0.50 & 0.005 & -5.2983 & -0.0265 & 28.0720 & 0.1404 \\
\hline Leguminosae & Tetrapleura tetraptera & 1.50 & 0.015 & -4.1997 & -0.0630 & 17.6375 & 0.2646 \\
\hline Meliaceae & $\begin{array}{l}\text { Entandrophragma } \\
\text { cylindricum }\end{array}$ & 0.50 & 0.005 & -5.2983 & -0.0265 & 28.0720 & 0.1404 \\
\hline Meliaceae & Guarea glomerulata & 0.50 & 0.005 & -5.2983 & -0.0265 & 28.0720 & 0.1404 \\
\hline Meliaceae & Khaya grandifolia & 0.50 & 0.005 & -5.2983 & -0.0265 & 28.0720 & 0.1404 \\
\hline Meliaceae & Khaya ivorensis & 0.05 & 0.005 & -5.2983 & -0.0265 & 28.0720 & 0.1404 \\
\hline Meliaceae & Lovoa trichiloides & 2.50 & 0.025 & -3.6889 & -0.0922 & 13.6080 & 0.3402 \\
\hline Meliaceae & Melicia excela & 1.50 & 0.015 & -4.1997 & -0.0630 & 17.6375 & 0.2646 \\
\hline Moracea & Ficus exasperate & 1.00 & 0.010 & -4.6052 & -0.0461 & 21.2079 & 0.2121 \\
\hline Moracea & Ficus umbelatum & 0.50 & 0.005 & -5.2983 & -0.0265 & 28.0720 & 0.1404 \\
\hline Moracea & Musanga cercropioides & 4.00 & 0.040 & -3.2189 & -0.1288 & 10.3613 & 0.4145 \\
\hline Moracea & Myrianthus arboreus & 1.00 & 0.010 & -4.6052 & -0.0461 & 21.2079 & 0.2121 \\
\hline Moracea & Treculia Africana & 1.00 & 0.010 & -4.6052 & -0.0461 & 21.2079 & 0.2121 \\
\hline Myristicaceae & Pycnanthus angolensis & 0.50 & 0.005 & -5.2983 & -0.0265 & 28.0720 & 0.1404 \\
\hline Ochnaceaea & Lophira alata & 1.00 & 0.010 & -4.6052 & -0.0461 & 21.2079 & 0.2121 \\
\hline
\end{tabular}


Agric. Biol. J. N. Am., 2012, 3(12): 486-492

\begin{tabular}{|l|l|l|l|l|l|l|l|}
\hline Palmae & Elaeis guineensis & 1.00 & 0.010 & -4.6052 & -0.0461 & 21.2079 & 0.2121 \\
\hline Papilionaceae & Baphia nitida & 1.00 & 0.010 & -4.6052 & -0.0461 & 21.2079 & 0.2121 \\
\hline Piperaceae & Nauclea diderrichii & 1.00 & 0.010 & -4.6052 & -0.0461 & 21.2079 & 0.2121 \\
\hline Polygalaceae & Carpolobia alba & 0.50 & 0.005 & -5.2983 & -0.0265 & 28.0720 & 0.1404 \\
\hline Polygalaceae & Carpolobia lutea & 0.50 & 0.005 & -5.2983 & -0.0265 & 28.0720 & 0.1404 \\
\hline Polygonaceae & Antiaris Africana & 4.00 & 0.040 & -3.2189 & -0.1288 & 10.3613 & 0.4145 \\
\hline Rubiaceae & Heinsia crinata & 2.50 & 0.025 & -3.6889 & -0.0922 & 13.6080 & 0.3402 \\
\hline Rubiaceae & Massularia acuminate & 1.50 & 0.015 & -4.1997 & -0.0630 & 17.6375 & 0.2646 \\
\hline Rubiaceae & Mytragyna ciliate & 0.50 & 0.005 & -5.2983 & -0.0265 & 28.0720 & 0.1404 \\
\hline Rubiaceae & Mitragyna stipulosa & 0.50 & 0.005 & -5.2983 & -0.0265 & 28.0720 & 0.1404 \\
\hline Rutaceae & $\begin{array}{l}\text { Zanthoxylum } \\
\text { xanthoxyloides }\end{array}$ & 0.50 & 0.005 & -5.2983 & -0.0265 & 28.0720 & 0.1404 \\
\hline Sapindaceae & Blighia sapida & 1.50 & 0.015 & -4.1997 & -0.0630 & 17.6375 & 0.2646 \\
\hline Sapotaceae & Baillonella toxisperma & 1.50 & 0.015 & -4.1997 & -0.0630 & 17.6375 & 0.2646 \\
\hline Sapotaceae & Chrysophyllum albidum & 1.50 & 0.015 & -4.1997 & -0.0630 & 17.6375 & 0.2646 \\
\hline Sterculiaceae & Cola acuminate & 0.50 & 0.005 & -5.2983 & -0.0265 & 28.0720 & 0.1404 \\
\hline Sterculiaceae & Cola gigangtea & 2.00 & 0.020 & -3.9120 & -0.0782 & 15.3037 & 0.3061 \\
\hline Sterculiaceae & Cola lepidota & 1.50 & 0.015 & -4.1997 & -0.0630 & 17.6375 & 0.2646 \\
\hline Sterculiaceae & Cola nitida & 0.50 & 0.005 & -5.2983 & -0.0265 & 28.0720 & 0.1404 \\
\hline Sterculiaceae & Sterculia tragacantha & 0.50 & 0.005 & -5.2983 & -0.0265 & 28.0720 & 0.1404 \\
\hline Sterculiaceae & Triplochiton scleroxylon & 1.50 & 0.015 & -4.1997 & -0.0630 & 17.6375 & 0.2646 \\
\hline Ulmaceae & Trema guineensis & 1.00 & 0.010 & -4.6052 & -0.0461 & 21.2079 & 0.2121 \\
\hline Verbanaceae & Vitex doniana & 1.00 & 0.010 & -4.6052 & -0.0461 & 21.2079 & 0.2121 \\
\hline & & & & & & & \\
\hline
\end{tabular}

$H_{1}=4.3459 ; \operatorname{Var}\left(H_{1}\right)=11.8661 ; H_{2}=4.0250 ; \operatorname{Var}\left(H_{2}\right)=7.7945 ; N_{l}=N_{2}=200 ; v(d f)=384 ; t_{c a l}=0.0724 ; t_{t a b}(0.05)=1.96$

\section{DISCUSSION}

The result shows that the diversity indices for the sanctuary $\left(H_{1}=4.3459\right)$ and communal forest $\left(H_{2}=\right.$ $4.0250)$ are not significantly different $(p>0.05)$ from each other. One hundred and two (102) tree species in 35 families were encountered in the sanctuary with Afzelia bipidensis $(\mathrm{RD}=5.00)$ as the only abundant species (Table 1). There was no record of frequent and occasional species while rare and threatened / endangered species were represented by fifty-two (52) and forty-nine (49) tree species respectively. In the communal forests, seventy-four (74) tree species were encountered in thirty-one (31) families (Table 2). Ceiba pentandra $(\mathrm{RD}=7.50)$ was the only abundant species in the communal forest while frequent species were represented by Harungana madagascariensis $(\mathrm{RD}=4.50)$, Albizia zygia $(\mathrm{RD}=$ 4.50), Musanga cercropioides ( $R D=4.00)$ and Antiaris africana $(\mathrm{RD}=4.00)$ as shown in Table 2 . Occasional species in the communal forests were absent. Rare species were represented by forty- two (42) tree species while threatened species were made up of twenty-seven (27) tree species. The dominance of Ceiba pentandra followed by Harungana madagascariensis, Albizia zygia and Musanga cecropioides as frequent species, indicated that the communal forests possessed some characteristics of secondary forest, possibly as a result of anthropogenic impacts occasioned by bush burning and agriculture. These are species which are common in a degraded forest as reported by Etukudo et al, (1994). Over-exploitation and utter conversion of forest ecosystems results in the decimation of tree species (Iroko et al.2008). The above analysis shows that there was likelihood of more tree extraction for timber and other uses in the communal forests than the protected area (sanctuary). Owing to this demand, some of these species become rare and threatened. The disappearance of many economically valued tree species across Nigeria is a well known phenomenon (Agbogidi, 2011; Adekunle and Akinlembola,, 2008; Sale et al., 2008; Oni et al., 2010). If forests are indiscriminately depleted, the various uses and roles associated with them would be lost.

CONCLUSION AND RECOMMENDATION: Different levels of disturbance have different effects on tree diversity in the study sites. Thus if the goal of management is to preserve biodiversity in the area, there is need to understand how diversity is impacted by different management strategies.

Reliable information on the status and trends of forest resources helps give decision makers the prospective necessary for orienting forestry policies and programs. Thus forest composition assessment of Afi Mountain Wildlife Sanctuary will serve as a valuable 
tool that will enable conservators and managers of the sanctuary to quantify tree diversity as well as providing information on numerical structure of species in the area of study.

The Cross River State Forestry Commission, which is saddled with the responsibility of managing the resources of the sanctuary, should design programmes that will create more awareness on the part of the people to see the need to protect flora and fauna species from being threatened. Domestication of indigenous tree species should be advocated both for poverty alleviation in the communal lands of the area, and for a balance to be maintained in the ecosystem (Jimoh and Haruna, 2007; Olufemi and Akinlosufu, 2006). Cultivation of edible and medicinal species in the communal forests should be encouraged by government and other stakeholders of Afi Mountain Wildlife Sanctuary and environs. This would reduce the rate of encroachment into the sanctuary for plant species exploitation for economic and medicinal reasons. Above all, more studies should be carried out to ascertain the level of disturbance and how this affects flora and fauna composition, distribution and abundance in Afi Mountain Wildlife Sanctuary and environs.

ACKNOWLEDGEMENTS: Our profound appreciation goes to the management and staff of the Cross River State Forestry Commission for allowing us to use their men and equipment for this research. We also thank the government of Cross River State, Nigeria for providing the necessary funds for the research to be carried out in this rugged terrain.

\section{REFERENCES}

Adekunle, V. A. J. and Akinlembola, O. (2008). Effect of Deforestation on Climate Change and Global Warming in Nigeria. In: Popoola, L (ed). Proceedings of the $32^{\text {nd }}$ Annual Conference of the Forestry Association of Nigeria (FAN) held in Umuahia, Abia State between $20^{\text {th }}$ and $24^{\text {th }}$ October, 2008. Pp 170-182.

Abgogidi, O. M. (2011). A Survey of the Economically Valued Forest Plant Species at the Proposed Site for Airport in Asaba, Delta State, Nigeria. Agriculture and Biology Journal of North America, 2012, 2(1): 143-149. Available from URL: http//www.scihub.org/ABJNA

Agbor, C. O. (2003). An Ecological Basis for the Management of Afi Mountain Wildlife Sanctuary, Cross River State. Unpublished PhD Thesis, Department of Wildlife and Fisheries Management, University of Ibadan. Pp. 119 210.

Bryant, D. M, Ducey, M. J., Innes, J. C., Lee, T. D., Ekert, R. T. and Zarin, D. J. (2005). Forest Community Analysis and the Point-Centered Quarter Method. Plant Ecology 175 (2): 193-203.

Conservation International (Cl) (2005). Biodiversity Hotspots. Cl Facts. Internet Communication (Cited 07-05-2012). Available from URL: http://www.fao.org/docrep/XO451E/XO45/el/htm.

Edet, D. I. (2010). Biodiversity Utilization Pattern in Afi Mountain Wildlife Sanctuary, Cross River State, Nigeria. Ph.D. Thesis, University of Ibadan, Ibadan, Nigeria.

Etukudo, I. G, Akpan-Ebe, I. N, Udofia, A. and Attah, V. I. (1994). Elements of Forestry. First Edition. Government Printer, Uyo, Nigeria. Pp. 9 - 16.

Iroko, O. A., Kareem, A. A., Adio, A. F. and Gbadebo, J. O. (2008). Impact of Human Activities on the Forest and their effects on Climate Change. In: Popoola, L (ed). Proceedings of the $32^{\text {nd }}$ Annual Conference of the Forestry Association of Nigeria (FAN) held in Umuahia, Abia State between $20^{\text {th }}$ and $24^{\text {th }}$ October, 2008. Pp 208214

Jayaraman, K. (1999). A Statistical Manual for Forestry Research. Food and Agriculture Organization of the United Nations (FAO) Regional Office for Asia and the Pacific, Bangkok. Pp. 191-196.

Jimoh, S. O. and Haruna, E. A. (2007). Contribution of NonTimber Forest Products to Household Food Security and Income around Onigambari Forest Reserve in Oyo State, Nigeria. Journal of Environmental Extension. 6:34-37.

Olufemi, B. and Akinlosufu, M. O. (2006). Contribution of Nonwood Forest Products to the Socio-economics of Rural Dwellers in Idanre, Ondo State, Nigeria. In: Popoola, L (ed). Proceedings of the $31^{\text {st }}$ Annual Conference of the Forestry Association of Nigeria (FAN) held in Makurdi, Benue State between $20^{\text {th }}$ and $25^{\text {th }}$ November, 2006. Pp 516-526.

Oni, Y. M., Onyekwelu, J. and Ajibufun, I. A. (2010). The Impact of Climate Change on Forest Resources. In:Onyekwelu, J., Adekunle, V. A. J. and Oke, D. O. (eds). Proceedings of the $2^{\text {nd }}$ Biennial National Conference of Forests and Forest Products Society of Nigeria held at the Federal University of Technology, Akure, Nigeria between $26^{\text {th }}$ and $29^{\text {th }}$ April, 2010. Pp 132-135

Sale, F. A., Samuel, D. and Ayo-Odifin, O. S. (2008). Effects of Climate Change on Biodiversity. In: Popoola, L (ed). Proceedings of the $32^{\text {nd }}$ Annual Conference of the Forestry Association of Nigeria (FAN) held in Umuahia, Abia State between $20^{\text {th }}$ and $24^{\text {th }}$ October, 2008. Pp 202207

Stirn, J. (1981). Manual of Methods in Aquatic Environment Research, Part 8. Ecological Assessment of Pollution Effects. Guidelines for the FAO / GFCM / UNEP Joint Coordinated Project on Pollution in the Mediterranean. Pp. 46 - 50.

Wikipedia - The Free Encyclopaedia (2012). World Data Base on Protected Areas. Internet Communication (Cited 0605-2012). Available from URL: http://www.wdpa.org. 